\title{
JUSTICIA TRANSICIONAL: DERECHO A LA VERDAD COMO CONSTRUCCIÓN DE FUTUROS
}

\author{
TRANSICIONAL JUSTICE: A RIGHT TO THE TRUTH \\ AS CONSTRUCTION OF FUTURE
}

Por: Aura María Echeverría*

*Filósofo y Abogado. Especialista en Ética y Filosofía Política, Magíster en Seguridad y Defensa Nacional Y Defensa Nacional, en Estudios Políticos. Candidato a Magíste en Conflicto Social y Construcción de Paz. Docente del Programa de Trabajo Social de la Corporación Universitaria Rafael Núñez. Investigadora del GruInvestigadora del Grupo de investigación Derecho Público. Inves tigadora del Grupo de Investigación Observatorio Desplazamiento Forzado de la Universidad de Cartagena.

Sidarrespondenciagena. mariaer@hotmail.com

Recibido: 11 de febrero de 2016 - Aprobado: 28 de junio de 2016

\section{RESUMEN}

El presente artículo hace parte del proyecto de investigación que tiene como objeto determinar la eficacia de las leyes que establecen la justicia transicional en Colombia, particularmente en algunos casos ocurridos en el departamento de Bolívar entre los años 1999 y 2001. Y dentro de los objetivos específicos del trabajo se propone conocer cada uno de los derechos correlacionales a la justicia transicional: verdad, justicia y reparación. En particular, el presente artículo es un avance del citado proyecto de investigación gira en torno a plantear que el conocer la verdad, vista ésta como construcción de la memoria histórica, puede facilitar los procesos de conciencia, colectividad, la sensibilización y el apoyo, hacia las víctimas y así la posibilidad de la reconciliación y de la construcción de paz en Colombia. El texto propone la siguiente estructura: i) definir lo que en el presente trabajo se debe entender como verdad, ya que es un concepto con varias acepciones y significados, ii) establecer como se relaciona la divulgación de esa memoria histórica con la construcción de futuro y transformación del conflicto en un contexto de complejidad creciente; iii) Posteriormente, plantear como se puede convertir esta en la base de un proceso de transformación del conflicto.

Palabras Claves: Justicia transicional, memoria, verdad, reparación, tranformación del conflicto.

\begin{abstract}
This article is part of a research project which aims to determine the effectiveness of laws establishing transitional justice in Colombia, particularly in some cases in the department of Bolivar between 1999 and 2001. The specific objective of the work aims to identify each of the correlational rights to transitional justice: truth, justice and reparation. In particular, this article is the progress of that research project which revolves around how knowing the truth, viewing this as the building of historical memory, can facilitate the processes of consciousness, community, advocacy and support, to victims and thus the possibility of reconciliation and peace building in Colombia. The text proposes the following structure: i) define what in this paper should be understood as true as it is a concept with several meanings and meanings, ii) establish the relationship of such historical memory with future construction and conflict transformation in a context of increasing complexity; iii) consider how this can become the basis of a process of conflict transformation.
\end{abstract}

Keywords: Conflict, efficiency, justice, post-conflict reparations, restitution, transitional justice, truth, victim, victimizer 


\section{Introducción}

1 l presente artículo se desprende de la investigación en ejecución que $\checkmark$ tiene como objetivo general "Determinar la eficacia de las leyes que establecen la justicia transicional en Colombia, particularmente en algunos casos ocurridos en el departamento de Bolívar entre los años 1999 y 2001". El interés de desarrollar el citado proyecto de investigación es la situación particular en la que se encuentra Colombia frente la aplicación de este tipo de justicia, con la particularidad de que este tipo de justicia se aplica en períodos donde el conflicto interno o la dictadura en los que han ocurrido graves violaciones a los derechos humanos ya han terminado, mientras que en Colombia, nos encontramos aún en una etapa de conflicto.

La investigación nos permitirá establecer un marco conceptual en cuanto a la definición de justicia transicional y de los derechos correlativos a ella, ocupándonos en especial del derecho a la verdad. De igual manera, las tendencias que existen en cuanto a un proceso de transición, las cuales dependen del conflicto mismo, de la sociedad, de las políticas implementadas y de las normas jurídicas que regulen el proceso. En este sentido el proyecto donde se origina el presente artículo, tienen en cuenta las experiencias de otros países que han logrado la transición, para conocer como han resuelto la tensión entre las obligaciones jurídicas frente a las víctimas, y las necesidades políticas para poder negociar el cese del conflicto con los grupos armados que llegaron a participar en él y de igual manera determinar que formas han sido eficaces, sin olvidar la importancia del contexto en el que se presentan.

El tipo de investigación que se va a desarrollar es de carácter aplicado, dado que busca implementar la teoría acerca de justicia transicional a los procesos que se adelantan en Colombia, particularmente en el departamento de Bolívar. Para alcanzar el objetivo general se realizará un estudio de casos, haciendo recolección de información que permita hacer el estudio intensivo de la situación actual en que se encuentra el trámite judicial y social por parte de las instituciones de Estado colombiano y un análisis de datos correspondientes a diversos puntos de vista o posiciones de distintos autores, que además pertenecen a diferentes áreas del conocimiento, tales como la filosofía, la sociología, la política y el derecho. Lo anterior supone la interdisciplinariedad de la investigación. 


\section{Resultados y discusión}

l l avance que se presenta en este artículo gira en torno a plantear que el conocer la verdad, vista esta como construcción de la memoria histórica, puede facilitar los procesos de conciencia, colectividad, la sensibilización y el apoyo, hacia las víctimas y así la posibilidad de la reconciliación y de la construcción de paz en Colombia. Es decir si las propuestas de transformación no se hace sobre el presupuesto de conocer la verdad de lo ocurrido, los hechos, las víctimas, los victimarios, el sufrimiento, el dolor, los muertos, los desaparecidos, las formas y sus crudezas, no es posible saber qué es lo que se va a reparar, qué es lo que se va a perdonar y a olvidar, no se podrá saber si hay justicia o impunidad, o si las medidas son excesivas o insuficientes.

Por su parte, en el caso de Colombia, conocemos juicios, y prejuicios sobre el proceso, y estos están construidos sobre falsas apreciaciones de la realidad o imaginarios que no son construidos con conocimiento de la verdad. Lo mismo ocurre, sobre el desconocimiento de las políticas, las leyes y sus reformas y así los medios de comunicación informan y construyen opinión.

Inicialmente, es necesario resaltar que si bien es claro que el conflicto es una constante de la vida humana, en el caso colombiano, parafraseando a Johan Galtung en su texto "Teoría de los conflictos", este ya se transcendió a sí mismo convirtiéndose en violencia que llegó hasta los límites de la deshumanización absoluta. Y con fundamento en lo afirmado por este autor, si no logramos conocer la complejidad del mismo, sus lógicas, sus actores y sus hechos, no podrá ser manejable y transformable. Es por ello que estudiando las teorías acerca de la verdad, la justicia, y la reconciliación, y haciendo un estudio de la experiencia en otros países, en los que la verdad tuvo gran valor, podremos afirmar que tan necesario es conocer la verdad de los hechos, hacer una edificación de la memoria histórica a fin de acerarnos a la reconciliación y así a la construcción de la paz.

Si lo que debemos conocer es la complejidad del conflicto, lo primero que debemos tener claro es la complejidad humana, y entonces sabremos que sobre los hechos de violencia existen un sinnúmero de historias, verdades que surgen sobre el conflicto, pero podremos tener claro que la finalidad es saber que el conocimiento de ellas nos puede llevar hacia la concientización y sensibilización y como consecuencia a la transformación del mismo y por ello a la posibilidad de la reconciliación y la reconstrucción de lo que se perdió. 
De forma general podemos señalar que si el origen de la conformación de organismos internacionales, la suscripción de tratados de derechos humanos, los procesos de construcción de paz y pedagogía social es el conocimiento, la conciencia sobre los hechos ocurridos en la Segunda Guerra Mundial, a partir de la llamada "nueva conciencia humanitaria" ${ }^{1}$, en Colombia al conocer los hechos, las víctimas y los responsables, se produzcan acciones que nos lleven a la construcción de la paz. De manera tal que si durante tanto tiempo hemos desarrollado una cultura de la violencia, podamos empezar a desarrollar una cultura de paz. El proceso sería, "si quieres paz, prepárate para la paz" en contraposición a la máxima que se sostuvo por muchos años, incluso actualmente "si quieres paz, prepárate para la guerra" (Galtung, 2003).

Para desarrollar el planteamiento de que la verdad puede ser el fundamento de la construcción de paz, debemos: i) definir lo que en el presente trabajo se debe entender como verdad, ya que es un concepto con varias acepciones y significados, ii) establecer como se relaciona la divulgación de esa memoria histórica con la construcción de futuro y transformación del conflicto en un contexto de complejidad creciente; iii) posteriormente, plantear como se puede convertir esta en la base de un proceso de transformación del conflicto.

\section{Concepto de verdad como memoria histórica}

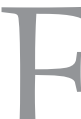

n el marco de los conflictos, la verdad puede ser entendida de diferentes maneras, entre las que podemos identificar dos, para los fines del presente ensayo, así: 1 . La verdad real y, 2. La verdad procesal ${ }^{2}$ y esta división da origen a lo que conocemos como: las comisiones de la verdad y las comisiones extrajudiciales de investigación. Para el objetivo de nuestro artículo trabajaremos con las comisiones de verdad, que en palabras de Priscila Hayner (2008) es una de las formas de rendir cuentas frente a los crímenes del pasado, en conjunto con la aplicación de la ley.

Uno de los objetivos de las comisiones de la verdad es hacer reconstrucción de los hechos, de los patrones de violencias, los actores, resaltando que la pretensión principal es la de clarificar, conocer los hechos y reconocer los abusos, las violaciones de derechos que ocurrieron en el pasado. Esto se hace con la intención de responder a las necesidades de las víctimas, si se puede contribuir a la justicia, y que exista un reconocimiento de responsabilidad de la instituciones y de los grupos armados. Es decir, las comisiones de verdad permiten "conocer los crímenes y crear un ambiente propicio para una reconciliación nacional" y "saber quiénes los cometieron y calcular el número
${ }^{1}$ Nueva conciencia humanitaria que surge al no aceptar que tantos crímenes fueron cometidos hasta el hecho de que en manos de los humanos podría estar el exterminio.

${ }^{2}$ En nuestro concepto la primera de ellas está más relacionada con la verdad como proceso de reconstrucción de los hechos, de una memoria de los relatos que debe conocer la sociedad, a manera de toma de conciencia, que ayude a los procesos de reparación picatrización, cicatrizacilin, perdon y reconciliación. La segunda más relacionada con el derecho a la justicia, a la reducción de la impunidad, a la aplicación de las sanciones. De igual manera, entendemos que las comisione de verdad "son las que responden a la necesidad de esclarecimiento de los hechos de violencia política en contextos de transición" (Ceballos, 2009), y las comisiones extrajudicomisiones extraju ciales de investigación "no solo le apuestan al esclarecimiento histórico, sino que por lo general se crean por la urgencia de otorgar impulso a la labor judicial en situaciones de dicial en situaciones de debilidad institucional son creadas durante el desarrollo del conflicto" (Ceballos, 2009). 
de víctimas"(Hayner, 2008). Igualmente, a través de ellas se materializa el carácter reparador, restaurador y simbólico que tiene la verdad, porque se presentan episodios de arrepentimiento y perdón entre las víctimas y los victimarios. Hechos que consideramos necesarios para iniciar el proceso de reconciliación nacional propio de las etapas de justicia transicional, que tienen como finalidad la instalación de la paz y la convivencia armónica.

Por lo antes dicho consideramos necesario que el resultado del proceso de recuperación de los hechos y de las verdades sea conocido por toda la sociedad, obedeciendo el hecho de que la reconciliación es de carácter nacional, ya que los efectos de los procesos recaen y ocurren sobre toda ella y en mucho el desarrollo y eficacia de los proceso depende de la conciencia que tengan todos los miembros de la comunidad sobre los mismos.

El concepto de verdad, en el contexto en que lo estamos aplicando, tiene múltiples acepciones y varios autores que buscan teorizarla, como por ejemplo: la Comisión Interamericana de Derechos Humanos (CIDH), que no solo las familias sino toda la sociedad en general tiene el derecho inalienable de conocer la verdad de lo ocurrido, así como las razones y circunstancias en las que aberrantes delitos llegaron a cometerse, a fin de evitar que esos hechos vuelvan a ocurrir en el futuro. (CIDH, 2000).

Estas conceptualizaciones además de plantear el hecho de que la sociedad tiene el derecho a conocer lo ocurrido, también plantea la verdad como ejercicio que hace posible la transformación del conflicto, evitando que se vuelvan a repetir los mismos hechos. En concepto de los organismos internacionales, la verdad no es solo un derecho sino una medida de satisfacción y de garantía de no repetición si cumple con los estándares, establecidos por los organismos internacionales. Este derecho que debe ser de la sociedad completa, en especial para facilitar los procesos de transición, y hacer una reconstrucción de la historia, en la que se expone la forma en que la sociedad narra el pasado, su relación con él, y su visión del mismo. En palabras de Siegel:

[m] uchos de nuestros entendimientos sociales constitutivos asumen forma narrativa $y$, muchas veces, estas narrativas incluyen historias acerca del pasado. Al contar historias acerca de un pasado común, un grupo se constituye a sí mismo como grupo, como sujeto colectivo con ciertas experiencias, expectativas, derechos, obligaciones y compromisos. Las historias que ayudan a forjar la identidad 
de un grupo también proveen estructuras para la comprensión ordinaria, marcos dentro de los cuales los miembros de una sociedad interpretan la experiencia y efectúan juicios normativos y positivos acerca de la misma. En suma, las narrativas acerca de la génesis de los arreglos sociales ayudan a constituir los grupos como sujetos colectivos y, al hacerlo, construyen sus intuiciones de sentido común acerca de la organización apropiada y actual de las relaciones sociales. (Botero, 2009).

Si se considera la necesidad de sanar las heridas del pasado para alcanzar una convivencia pacífica y un nuevo pacto social para avanzar hacia la reconciliación nacional, estos procesos de reconstrucción del pasado y de publicidad del mismo, adquieren incluso una fuerza histórica esencial. Por ello afirmamos que del ejercicio de reconstrucción de la verdad deben hacer parte todos los actores del conflicto, los visibles y los no visibles. Es decir, consideramos que la memoria histórica, está conformada por el recuento de varias memorias individuales, la de cada uno de los actores como sujetos, memorias sociales, de los grupos a los que pertenecen los sujetos, y el componente del pasado, memoria histórica. Lo anterior evidencia no solo la gran carga de subjetividad que tiene esta reconstrucción de la verdad, sino el alto grado de complejidad ${ }^{3}$ en ella.

Finalmente, para concluir este apartado parafraseamos lo sostenido por Leslie Sequeira (2009), en su dosier sobre la recuperación de la memoria histórica y sus dilemas, reconstruir la memoria debe servir para motivar la convivencia y la paz, fortalecer la democracia, luchar, promover y defender los derechos humanos; debe recordarse que recuperar la memoria histórica es una cuestión de derechos humanos.

\section{Complejidad y verdad: Divulgación y construcción de futuro}

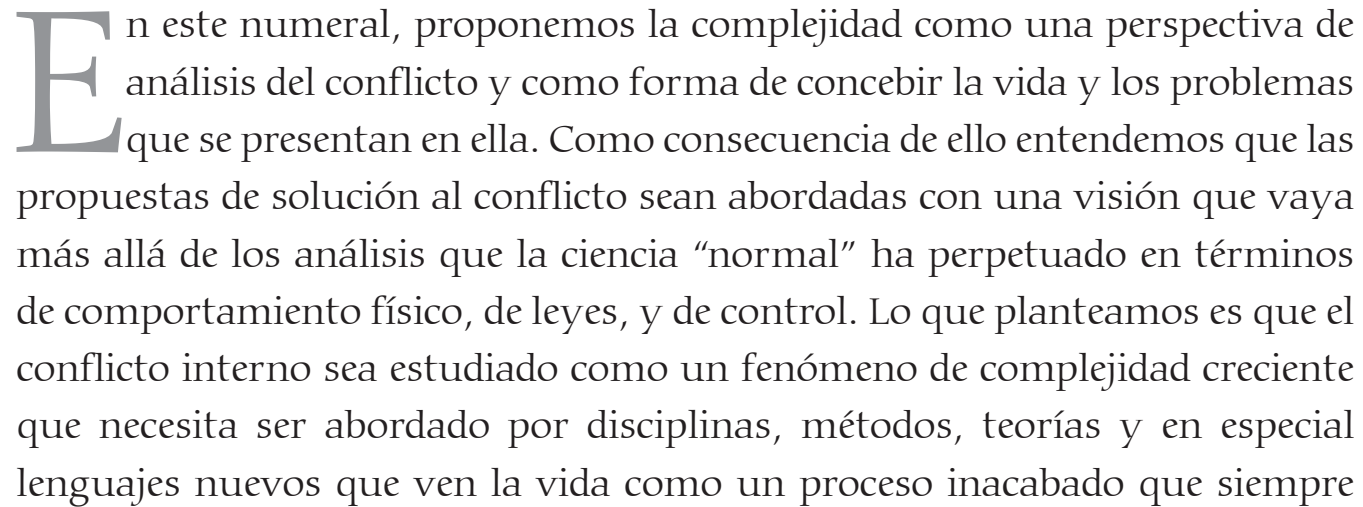

${ }^{3}$ Entendiendo complejidad como una forma de asumir la vida y en particular la perspectiva como deben asutiva como deben asu-
mirse los fenómenos sociales. 
está en movimiento e indeterminación. (Maldonado, 2012) Es decir, nuestra propuesta es que la compresión del conflicto armado y sus alternativas de transformación sea intentada desde la lógica de la no linealidad, desde el cruce, el diálogo, la cooperación de disciplinas diferentes.

Para poder comprender fenómenos de este tipo debemos tener claro que dentro de los objetos y fenómenos complejos, la vida es el de mayor complejidad, parafraseando a Estanislao Zuleta (1969) y en particular, la complejidad de la situación en Colombia aumenta ya que el conflicto como sus hechos y las miradas acerca el mismo están en movimiento y construcción permanente, y por tanto no se conducen por la línea del tiempo lineal, sino que a medida que avanzamos se producen cambios, nuevos sentimientos, otras visiones. Como consecuencia, las posibilidades de mirar lo que ha ocurrido y las formas de transformarlo dependen de que podamos aceptar que la complejidad que entraña el contexto lo convertirá siempre en algo indeterminado e incompleto, ya que no obedece a una única lógica, y si intentamos reducirla estaríamos dejando de lado una cantidad de matices y de posibilidades que son igualmente valiosas.

Para ser fieles a lo expuesto, entendemos el concepto de verdad como una noción relativa a mundos posibles, entonces para abordar acciones de construcciones de verdad, paz, pedagogía social, planteamos hacerlo desde la propuesta del profesor Maldonado (2012), de la racionalidad colectiva y de la acción colectiva, en la que el colectivo actúa como un organismo y no de una suma de individuos. Es decir, debemos como sociedad comprendernos como un sujeto social, que tiene una vida, espacios de deliberación y acción colectiva de la que debe beneficiarse el organismo colectivo y no el individuo, negando así la posibilidad del egoísmo del individualismo.

En esto radica el argumento central del presente artículo, en que para poder conocer, tener conciencia del colectivo del que hacemos parte, debemos conocer la verdad de lo ocurrido, y esto depende de la reconstrucción que se haga y de la divulgación de la misma como memoria histórica, construida con el mayor número de subjetividades posibles, como base para poder tener un imaginario colectivo que será el fundamento de lo que hemos sido y que nos marcará lo que podemos ser, lo que queremos ser, lo que queremos construir y las posibilidades para llegar a ello. Solo si conocemos podremos garantizar que la sociedad, en términos de imaginarios colectivos, puede generar nuevos lenguajes para designar una nueva concepción del mundo, de país, de sociedad. 
Así, desde los planteamientos de las ciencias de la complejidad, es el lenguaje el primer objeto que permite la existencia de los colectivos y organizaciones sociales, ya que es este el encargado de generar diálogos, acuerdos, el sentido y las formas de interacción entre los miembros de la organización. Es el lenguaje el que da la posibilidad de que el discurso del colectivo sea inclusivo o exclusivo, es alrededor de él que se genera disenso o consenso, por tanto, es el lenguaje el elemento que permite que la articulación de los sujetos pueda hacerse de una manera distinta a la tradicional, para lo que es necesario uno nuevo.

Y esa manera tradicional o histórica, solo la podemos conocer y tener conciencia de ella a través de las narraciones de los actores visibles e invisibles del conflicto, lo que nos va a permitir saber cuál es el lenguaje que vamos a de-construir para a partir de allí construir uno. En caso de no hacerlo estaríamos consolidando y perpetuando la realidad existente, en palabras de diferentes autores, se estaría normalizando las formas de violencia que se presentan en el conflicto colombiano. Cosa que ha venido ocurriendo y que por desconocimiento permitimos y reproducimos dando paso a los diferentes tipo de violencia, directa, estructural las cuales se perpetúan en forma de violencia cultural.

En su texto Alejandro Peñuela (2009) señala que: "Pero solo en el acto del análisis, de la verbalización, el sujeto representa, recrea, asigna imágenes, valores simbólicos a lo que se encuentra dentro de su campo de percepción en función de su historia como un sujeto in-formado". De igual manera, y en forma más contundente, el profesor Gabriel Vélez Cuartas plantea porque el lenguaje es el objeto más importante si queremos generar nuevas formas de relaciones sociales de la siguiente manera: "Las relaciones sociales desde este punto de vista, sólo pueden ser explicadas a partir del sentido construido por los sujetos, reflejado en sus expresiones lingüísticas". (Vélez, 2011)

Entonces, la memoria histórica y las alternativas de transformación del conflicto deben ser el resultado del ejercicio que se hace con una sociedad que tiene conocimiento de lo que ha ocurrido en su territorio, la crudeza y la crueldad con la que ocurrió. Del ejercicio que se hace con una mirada compleja e integradora de varias miradas de lo que ha ocurrido, es decir que de valor a cada una de las voces que narran los hechos. Ejercicio donde además la sociedad que escucha y que narra es plenamente consciente que está ejerciendo su derecho y su obligación. Consideramos que este es el punto de partida para que la sociedad colombiana sea partícipe de nuevas dinámicas 
${ }^{4}$ Dicho método propone unos elementos básicos, así:

- Superar el conflicto con la finalidad de que todas las partes alcancen sus objetivos.

- Hacer, crear las condiciones para que los aspectos transformativos sean dominantes.

- Observar el dialogo como el método más adecuado para llevar a cabo esta acción. Diálogos transforión. Diálogos transforma-

Empatía, creatividad y no violencia.

Considerar a los actores como partes iguales. La transformación va más allá de las asimetrías sociales.

Suponer el conocimiento de todas las circunstancias que rodean al conflicto, as como los orígenes de la violencia.

- Suponer la presencia de una tercera persona, el trabajador de la paz, que actúe fundamentalmente como moderador entre las partes implicadas.

Implicar las nociones de las 3R (Reconciliación, Reconstrucción, Resolución).

- Implicar los niveles micro, meso, macro y mega de la condición humana.

- Tiene como sus elementos metodológicos: no violencia, creatividad y empatía.

Tiene incorporado el principio no violento de la reversibilidad. sociales, culturales y de vida que modifiquen las relaciones, las identidades, y las prácticas violentas actuales.

Pero incluso para saber que son nuevas, debemos saber qué el conflicto fijó formas de vida, imaginarios de los habitantes, de las organizaciones, y un lenguaje propio, además debemos identificar cuáles son para poder iniciar con el proceso de deconstrucción y construcción de la cultura de violencia. El proceso pedagógico se traduce en la expresión "si quieres paz, prepárate para la paz" generando una ruptura en la comprensión de la realidad colombiana y así la transformación de las relaciones sociales que ha fijado el conflicto, además ampliar la posibilidad de los futuros, nos pueden llevar a concebir diferentes posibilidades de paz, vista esta como "ayuda mutua para el desarrollo". Definición planteada por Kropotkim en su texto Mutual aid, citado por Johan Galtung (Galtung 2003), que encaja perfectamente en la mirada compleja del tema del conflicto y de la paz, en ella no se describe que es el desarrollo, lo que hace posible que sea definido por el antidestino (Núñez, 2007).

\section{Proceso de transformación del conflicto desde la base del conocimiento en el contexto colombiano}

\section{Transcendencia y transformación del conflicto según}

\section{la teoría de J. Galtung}

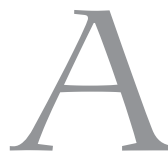
corde con los expuesto en el acápite anterior, en donde argumentamos que es necesario tener conocimiento de los hechos ocurridos, a través de la reconstrucción de la memoria histórica y su divulgación, con el fin de construir conciencia y sensibilización en la sociedad, y así iniciar la transformación del conflicto a través de los cambio de lenguaje y representaciones de la colectividad, y de los imaginarios colectivos actuales, para así promover los futuros, pasamos a plantear como esta propuesta se puede convertir en la base de un proceso pedagógico de cultura de paz.

La construcción de la memoria histórica, y su relación con el derecho a la verdad, lo complejo de este proceso y los beneficios que encontramos en el ejercicio de que la sociedad conozca lo ocurrido nos permite afianzar una propuesta de Johan Galtung (2003), en particular su método Trancend, ${ }^{4}$ que tiene como objetivo transcender y transformar los conflictos, a través de la reconstrucción, reconciliación y resolución de los mismos, no como la construcción de algo nuevo, sino como hacer resurgir las habilidades olvidadas por el conflicto. De igual forma para el autor "una teoría de conflictos deberá 
abordar todas las realidades que constituyen la condición multinivel de la especie humana". ${ }^{5}$

Con la aplicación de este método la sociedad debe construir formas de superar los traumas, los hechos de violencia y sus orígenes, proponiéndose metas comunes a la colectividad. Esas metas solo pueden establecerse a partir de conocer los límites que impuso el conflicto, y del diálogo entre los actores del mismo y de la sociedad en general. El ejercicio debe empezar por las experiencias micro, y seguir por grupos que inicien con la transformación de lo ocurrido. En palabras del autor, estas narraciones deben ser divulgadas con el objeto de que vayan propiciando modificaciones en la realidad y produciendo nuevos imaginarios en el colectivo. También afirma que transcender el conflicto es ir más allá de él, más allá de sus fundamentos, los cuales fueron conocidos a través del diálogo.

\section{Colombia y la posibilidad de transformación del conflicto}

$\mathrm{P}$ ara el caso colombiano no es diferente, toda la sociedad debe conocer los hechos y los actores, particularmente el sufrimiento y las consecuencias niveles de deshumanización de la guerra. En el momento de transición que nos encontramos, se deben conocer las narraciones que hay acerca del conflicto, los avances y los acuerdos que se han logrado y la situación que siguen viviendo todas la zonas del país, ya que no se tiene conciencia de las necesidades ni de las carencias en la que se encuentra esa gran parte de la sociedad. Teniendo en cuenta que muchas de las circunstancias anteriores son desconocidas por la sociedad, los proyectos de reconciliación, reconstrucción del tejido social son poco viables, y en muchos caso, particularmente en el caso de los desplazados y despojados estos son revictimizados, causando más sufrimiento.

En la aplicación de las leyes y decretos ${ }^{6}$ marcos de la justicia transicional se han conocido declaraciones sobre los hechos, y algunos pocos testimonios de las víctimas que han intentado narrar su sufrimiento. De igual manera, se han creado instancias como la Comisión Nacional de Reparación y Reconciliación - CNRR y el Centro Nacional de Memoria Histórica, que han generado unos valiosísimos documentos que reconstruyen los hechos ocurridos en unas zonas del país, los cuales demuestran un esfuerzo valioso en la reconstrucción de la memoria, también es cierto que estos no se conocen masivamente.

${ }^{5}$ Los niveles a los que hace referencia el autor son los siguientes:

- Micro: Es el nivel interpersonal, el que solo implica al individuo. Ya que se entiende a la persona como sistema complejo.

- Meso: Es cuando el individuo interactúa en grupos, ya sean más de dos personas.

- Macro: Es el nivel que implica a la sociedad o al Estado, es decir, cuando los que interactúan son grupos que constituyen a la sociedad.

- Mega: Cuando las sociedades interactúan como civilizaciones y regiozaciones y regiones, constituyendo ractúa en el sistema planetario.

${ }^{6}$ Ley 975 de 2005, Ley 1448 de 2011, y sus decretos reglamentarios, entre otros. 
Los textos han sido publicados, los procesos de visibilización de las víctimas han sido documentados, pero ello resulta insuficiente ya que todavía la sociedad no tiene conciencia sobre su participación en la construcción de la verdad, en los ejercicios de memoria histórica, ni siquiera se visualiza como un sujeto colectivo que tiene la obligación de aportar al proceso de negociación, de cese y de transformación del conflicto. Esto ha originado una percepción y unas representaciones e imaginarios que son poco positivos frente a algunas acciones y mecanismos para la transformación del conflicto, como son la reparación de las víctimas y la negociación del cese de hechos violentos y reintegración de los victimarios, los acuerdos para la desmovilización de los grupos armados al margen de la ley. Una de la claras consecuencias es que la sociedad no ha pensado el proceso desde su nivel de interacción meso, macro y mega, sino como un proceso alejado de la realidad de la gran mayoría de la población, en especial de la urbana.

La sociedad no tiene conciencia, ni conocimiento del proceso de transición que atravesamos, y cómo es posible si aún nos encontramos en medio del conflicto, de suyo entonces no se conoce que en el marco de estas acciones se establecen mecanismos y garantías tanto para las víctimas como para los victimarios en aras de lograr la armonía y la reconciliación nacional y por ello existen reacciones de apatía, poca solidaridad y reacciones adversas tanto a las medidas, en especial las de justicia y penas alternativas, como a las acciones que adelantan las víctimas, y el aumento de tributos para poder indemnizar y atender a los actores directos de los hechos.

En consonancia con lo anterior, no toda la sociedad sabe que se encuentra en un proceso de transición y transformación del conflicto, por tanto se mantiene al margen de lo que ocurre no aporta a la reconstrucción del país, perpetuando las estructuras de violencia que ya ha construido la guerra. Naturalizando las diferentes formas de violencia sin que seamos conscientes de ellas. Para contrarrestar esto, y desarrollar propuestas de transformación de la realidad se deben fortalecer las acciones de reconstrucción de la memoria y hacer ejercicios pedagógicos con ellas, mostrando la verdad de lo ocurrido y vincular a estas estrategias a los medios de comunicación, las escuelas, las universidades, los organismos encargados de esta función, con el fin de que se genere un clima propicio para la generación de relaciones sociales nuevas que alienten un sentido de sensibilidad social para con la transformación del conflicto. Lo anterior, ya que hasta que no seamos conscientes de lo que hemos vivido como 
colectividad, como organismo social y sigamos en el individualismo egoísta en el que muchos se encuentran, no será posible generar unas nuevas dinámicas sociales, culturales y de vida que modifiquen las relaciones, los imaginarios y esas mismas prácticas sociales violentas en las que nos encontramos y que se han arraigado en la cultura colombiana.

\section{Conclusiones}

I finalizar debemos resaltar que el conflicto es una constante en la
vida humana, en el caso colombiano, la violencia transcendió los
límites de la deshumanización absoluta haciéndose cada vez más compleja, al involucrar más actores, más hechos, al extenderse en el tiempo y el espacio. Esto hace que para que sea posible abordarlo y transformarlo debe hacerse desde una visión que integre todas las dimensiones y versiones que existen acerca de lo ocurrido.

Teniendo en cuenta esta afirmación podemos rescatar el valor que cobra la verdad, como derecho y obligación de todos los actores directos e indirectos del conflicto. Ya que solo al conocer lo ocurrido es posible dimensionar el nivel de complejidad que abarca la realidad que se pretende transformar. Es por esta razón que planteamos que el ejercicio del derecho a la verdad, debe permitir hacer una edificación de la memoria histórica y a través de ello acercarnos a la posibilidad de reconciliación y de construcción de paz, sobre unas bases sólidas.

Una de las capacidades que se pierde con ocasión del conflicto es la de sensibilización, la de solidaridad y de empatía con el otro, o de la posibilidad de ser un sujeto colectivo. Y son precisamente estas, las que permiten desarrollar la conciencia de lo ocurrido, de los niveles de sufrimiento, de los daños que causa la violencia y así generar tipos de participación organizada que aporten a la cultura de paz, que se acompase con lo que se ha denominado "nueva conciencia humanitaria" llevándonos a deconstruir y reconstruir las formas de vida que la cultura de la violencia ha fijado, planteando un nuevo lenguaje que nos permita denominar esa nueva forma de vida e iniciar procesos de cambio que sean positivos.

Lo anterior es el fundamento de lo que afirmamos en este artículo, en relación con que el proceso debe ser público en cumplimiento del derecho y la obligación que tiene la sociedad de conocer lo ocurrido, para saber qué es lo que va a superar, a perdonar, a reconstruir, saber qué fue lo que se perdió y lo más 
importante de todo qué es lo que quiere, hacia dónde va y qué es lo que no va a permitir que vuelva a ocurrir.

De igual manera, una herramienta metodológica válida que aborda perfectamente nuestra posición de la mirada compleja de la realidad y de la importancia de la divulgación de la verdad, es la propuesta de Galtung denominado Método Trancend, que tiene como finalidad transcender y transformar los conflictos, a través de la reconstrucción, reconciliación y resolución de los mismos, no como la construcción de algo nuevo, sino como hacer resurgir las habilidades olvidadas por el conflicto haciendo ejercicio que tengan en cuenta las realidades que constituyen la condición multinivel de la especie humana, y la multidiscipinariedad de ello.

La posibilidad de transformación positiva del conflicto, radica en que este como un fenómeno constante de la vida se asuma como un proceso inacabado que siempre está en movimiento e indeterminación, como un fenómeno social que abarca la realidad de toda la sociedad, que en el caso específico de Colombia ha atravesado las relaciones sociales de muchas generaciones. Esto hace que para encontrar planteamientos que nos lleven a construir paz, a desarrollar acciones de pedagogía social que contengan las miradas plurales acerca del conflicto, sus actores y consecuencias, cambios de lenguaje, de imaginarios sociales, que amplíen la posibilidad de futuros que incluyan el sujeto colectivo como organismo y no como una suma de individuos.

Es decir, la transformación positiva del conflicto debe ser una dinámica social, cultural y de vida que modifiquen las relaciones, los imaginarios y esas mismas prácticas sociales violentas en las que nos encontramos y que se han arraigado en la cultura colombiana sobre las cuales debemos tener conocimiento y conciencia, a través del ejercicio de la verdad como un derecho y como una obligación si se quieren construir futuros posibles de paz.

\section{BIBLIOGRAFÍA}

Botero, Catalina. Uprinmy, Rodrigo. Saffon, María Paula. Restrepo, Esteban. (2006). ¿Justicia transicional sin transición? Verdad, justicia y reparación para Colombia. Bogotá. Antropos.

Ceballos, Marcela. (2009). Comisiones de la verdad en Guatemala, El Salvador y Sudáfrica, perspectivas para Colombia. Bogotá. La Carreta.

Comisión Interamericana de Derechos Humanos. (2000). Monseñor Óscar Arnulfo Romero y Galdámez V. El Salvador. Caso No. 11.481. Informe No. 37/00. 
Corte IDH. (2002). Caso Bámaca Velásquez. Reparaciones. Sentencia de febrero 22 de 2002. Serie C No. 91.

Corte IDH. (2002a). Caso Trujillo Oroza. Reparaciones. Sentencia de febrero 27 de 2002. Serie C No. 92 .

Corte IDH. (2003). Caso Myrna Mack Chang. Sentencia de noviembre 25 de 2003. Serie C No. 101.

Galtung, Johan. (2003) Paz por medios pacíficos. Paz y conflicto, desarrollo y civilización. Bilbao, Gernika Gogoratuz.

Hayner, Priscilla. (2008). Verdades inmemorables. México. Fondo de Cultura Económica.

Maldonado, Carlos Eduardo. (2012). Significado e impacto de las ciencias de la complejidad. Disponible en: http://Www.carlosmaldonado.org/articulos/EL\%20PROBLEMA\%20DE\%20UNA\%20 TEOR\%CDA\%20GENERAL\%20DE\%2OLA\%20COMPLEJIDAD.pdf.

Núñez, Violeta. (2007). Pedagogía social: un lugar para la educación frente a la asignación social de los destinos. Disponible en línea: http://www.porlainclusion.educ.ar/documentos/Violeta_N_ Pedagogia_Social.pdf

Peñuela, Alejandro \& Álvarez, Luis Guillermo. (2009). Colectivo: implicaciones sociales. Una aproximación psicológica a las agendas de información. Disponible en línea: http://www. razonypalabra.org.mx/anteriores/n26/lpenuela.html

Sequeira, Leslie. (2009). La recuperación de la memoria histórica y sus dilemas. Disponible en línea: http://www.irenees.net/bdf_fiche-analyse-943_es.html noviembre de 2013.

Vélez Cuartas, Gabriel. (2011). El cambio en las redes: una aproximación a las relaciones sociales desde el lenguaje, la representación y la institucionalización. Disponible en línea: http://revista-redes. rediris.es/html-vol10/vol10_6.htm

Zuleta, Estanislao. (1969). El elogio de la dificultad. Disponible en: http://www.elabedul.net/ Articulos/el_elogio_de_la_dificultad.php 